\title{
Editorial: Plant-Microbe-Insect Interaction: Source for Bio-fertilizers, Bio-medicines and Agent Research
}

\author{
Gero Benckiser ${ }^{1 *}$, Anton Hartmann ${ }^{2}$, Krishnamurthy Kumar ${ }^{3}$ and Bernd Honermeier ${ }^{4}$ \\ ${ }^{1}$ Applied Microbiology, Justus Liebig Universität Gießen, Giessen, Germany, ${ }^{2}$ Deutsches Forschungszentrum für Gesundheit \\ und Umwelt, Helmholtz Zentrum München, Munich, Germany, ${ }^{3}$ Department of Agricultural Microbiology, Tamil Nadu \\ Agricultural University, Coimbatore, India, ${ }^{4}$ Department of Agronomy and Plant Breeding, Justus Liebig Universität Gießen, \\ Giessen, Germany
}

Keywords: tripartite sebacinalean symbiosis, MATI regulation, virulence reduction, antimicrobial activity, nematode subpressing

\section{Editorial on the Research Topic}

Plant-Microbe-Insect Interaction: Source for Bio-fertilizers, Bio-medicines and Agent Research

The plethora of taxonomically, phylogenetically, and metabolically diverse soil- microbes, plants and animals is responsible for the dynamic observed in ecosystems. Especially the above- and below-ground species flexibility that drives biogeochemical processes is not well understood but is of great significance for food production networks and yield stability (Benckiser, 1997; Benckiser and Schnell, 2007)

Ecosystem management and agricultural praxis have until now been largely conducted in

OPEN ACCESS

Edited and reviewed by: Victor Flors,

Universitat Jaume I, Spain

*Correspondence:

Gero Benckiser

gero.benckiser@umwelt.uni-giessen.de

Specialty section:

This article was submitted to

Plant Microbe Interactions,

a section of the journal

Frontiers in Plant Science

Received: 05 April 2018

Accepted: 11 June 2018

Published: 28 June 2018

Citation:

Benckiser G, Hartmann A, Kumar K and Honermeier B (2018) Editorial:

Plant-Microbe-Insect Interaction:

Source for Bio-fertilizers,

Bio-medicines and Agent Research.

Front. Plant Sci. 9:931.

doi: 10.3389/fp/s.2018.00931 isolation but the recent interconnection of inextricably various disciplines has now given rise to a more holistic approach. This Frontiers' e-book on "Plant-Microbe-Insect Interaction: Source for Bio-fertilizers, Bio-medicines and Agent for Research" presents 42 contributions covering a broad spectrum of modern physico-chemical, biochemical, molecular biology and agronomy techniques with the aim to provide perspectives on how a better productivity, health and fitness could be achieved. The focus lies in the long term adapted composition and functioning of microbe-insect interactions, which should be brought in line with agricultural productivities.

In modeling of biological networks and system biology approaches Kumar et al. are introducing and giving insights into Indian rice landrace diversity Imam et al. as well as Saxena et al. and Wei et al. on anthracnose management in chilli and wide range of cruciferous crops. The cofactor vitamin B1 plays an important role in metabolic reactions of all living organisms and it is not surprising that thiamine biosynthesis of endophytic Henderosonia toruloidea colonizing palm oil seedlings improves their growth (Kamarudin et al.). In reports on two- or tripartite mutualistic symbioses Hashem et al. address the symbiosis between arbuscular mycorrhizal fungi (AMF) and "Formosa" azalea, Azalea gerrardii, Wei et al. describe how the mycorrhizal fungus Oidiodendron maius positively influences the growth of Rhododendron fortunei Lindl., Guo et al. describe fitness rise in a cooperation of Rhizobium radiobacter, Piriformospora and cereal crops, and Sharma et al. found salt stress adaptation of peanuts in the presence of halotolerant, endophytic, plant growth promoting rhizobacteria (PGPR). The endophyte Acidovorax radicis $\mathrm{N} 35$, a major producer of $N$-acyl homoserine lactone (AHL) type, favors growth of barley (Han et al.), the medicinal plant Hypericum perforatum shows a remarkable activity against bacterial and fungal pathogens (Egamberdieva et al.), and bacteria identified by pyrosequencing combined with qPCR effectively suppress Fusarium wilt in the Chinese medicinal plant Pseudostellariae heterophylla (Wu et al.). 
A selective pressure niche to plant growth promoting bacteria (PGPB) evolution is the Brazilian Iron Quadrangle (IQ) soil (Felestrino et al.). IQ soil is a specific reservoir for PGPB species useable in reforestation of antropized soil and one of those could be Pseudomonas aeruginosa PM12, which induces systemic resistance in tomato against Fusarium wilt (Fatima and Anjum). Phenolic, allelochemically acting acids apparently mediate shifts in soil rhizosphere microbial flora, which inter alia ameliorate tomato Fusarium wilt resistance in mono-cropping systems of the medicinal plant Radix pseudostellariae (Wu et al.).

The TIFY domain of poplar species contains approximately 36 conserved amino acids (Xia et al.). The forming core motif $\mathrm{TIF}[\mathrm{F} / \mathrm{Y}] \mathrm{XG}$ plays a role in poplar growth, tissue development, and defense regulation. Fungus-like growing rhizosphere actinomycetes are known to promote growth and yield enhancement in wheat crops through the plant growth hormone indole acetic acid (IAA) and among rhizospheric IAA producers the most active and good candidates for developing biofertilizers are Streptomyces nobilis, Streptomyces kunmingenesis, and Streptomyces enissocaesilis (Anwar et al.). A candidate for agroactive compound development is also Streptomyces hydrogenans, (Manhas and Kaur). This non-cytotoxic, non-mutagenic, 10-(2,2-dimethyl-cyclohexyl)-6,9-dihydroxy-4,9-dimethyl-dec2 -enoic acid methyl ester producing actinomycete causes severe morphological alterations in Alternaria brassicicola (Kaur et al.).

Besides Streptomyces species, Bacillus cereus AR156 is also a suppressing agent (Nie et al.). AR156-treated Arabidopsis plants activate MAPK signaling and FRK1/WRKY53 gene expression, both of which are involved in pathogen associated molecular pattern (PAMP)-triggered immunity (PTI) and suppress Pseudomonas syringae pv. tomato DC3000 (Pst DC3000). The strength of induced systemic resistance (ISR) against Botrytis cinerea due to these bacteria remains unknown (Nie et al.). A common bacterial rice pathogen is the virulent, biofilm forming Xanthomonas oryzae pv. oryzae (Singh et al.). With a combined application of Thyme oil (THY) and conventional bactericides the motA, motB, flgE, rpfF gene activity can be reduced, extracellular endoglucanase, xylanase, cellobiosidase, and polygalacturonase genes are expressed, the bactericide dose can be lowered, and disease progression be diminished.

An antioxidative amino acid is the histidine-derived, sulfurcontaining, non-proteinogenic ergothioneine (EGT) that adds to phyllospheric fitness (Alamgir et al.). M. aquaticum strain 22A produces high amounts of EGT. It has been isolated from a moss and has been only poorly studied. This is also the case for the facultative methylotrophic, pink pigmented bacteria (PPFM), identified as Delftia lacustris, Bacillus subtilis, or B. cereus by $16 S$ rRNA gene sequence analysis. They are directly antagonistic against fungal pathogens of tomato and regarded as biocontrol agents (Janahiraman et al.). Out of 217 rhizobacterial isolates obtained from six different Indian Assam tea estates, Dutta et al. identified Enterobacter lignolyticus, Bacillus pseudomycoides, Burkholderia sp., or $P$. aeruginosa by partial 16S rRNA gene sequence analysis and as bio-fertilizer candidates for tea crops. Tamilselvi et al. identified Brevibacterium sp. SOTI06 as calcite dissolving bacterium, Krithika and Balachandar identified Bacillus sp. or Enterobacter cloacae as zinc (Zn) solubilizing bacteria, and Shakeel et al. reports that E. cloacae releases under iron- sufficient and-deficient conditions $\mathrm{Zn}$ regulated transporters and iron $(\mathrm{Fe})$-regulated transporterlike proteins (ZIP) into the rice rhizosphere. Islam et al. assessed by quantitative real-time reverse transcription PCR that the 234 isolates obtained from the roots of basmati385 and basmati super rice varieties translocate $\mathrm{Zn}$ toward grains and suppress economically important rice pathogens as Pyricularia oryzae and Fusarium moniliforme. In Bangladesh, Pseudomonas stutzeri, B. subtilis, Stenotrophomonas maltophilia, Bacillus amyloliquefaciens, present in cucumber rhizosphere and identified by phylogenetic $16 \mathrm{~S}$ rRNA sequences, characteristically change the morphology of Phytophthora capsici hyphae growing toward PGPR colonies and from B. amyloliquefaciens (SN13) it is known that it prolongs stress tolerance in rice and acts as biocontrol agent against Rhizoctonia solani (Srivastava et al.). The virulence of fruit rot fungi during cranberry fruit development influences the release of ROS suppressive compounds such as benzoic and quinic acids (Tadych et al.) and intercropping can minimize the success of pea weevil larvae (Bruchus pisorum L.; Teshome et al.). Some genotypes have the capacity to obstruct pea weevil larval entry into developing seeds [neoplastic $(N p)$ genotypes] of $P$. sativum and intercropping $N p$ genotypes with other crops such as sorghum and maize can facilitate neoplasm formation. Insect-plantbacteria interaction is also the subject of Harun-Or-Rashid et al., who studied the widespread yield loss of many crops caused by the green peach aphid, a most destructive, plant sap sucking and plant viruses transmitting insect pest. Harun-Or-Rashid et al. found that Bacillus velezensis YC7010 which induces systemic resistance against bacterial and fungal pathogens of rice, can also induce systemic resistance against the green peach aphid (GPA), Myzus persicae, puncturing Arabidopsis leaves. B. velezensis significantly reduces setting, feeding and reproduction of GPA on Arabidopsis leaves by strongly inducing the senescencepromoting gene PHYTOALEXIN DEFICIENT4 (PAD4) while suppressing BOTRYTIS-INDUCED KINASE1 (BIK1). Another important soil-borne pathogen that affects field crops worldwide is Heterodera avenae. Trichoderma longibrachiatum has the ability to control $H$. avenae by surrounding the eggs completely with dense mycelia, lysing the content at late egg stage, and inducing a defense response in wheat plants (Zhang et al.). Insect-plant-bacteria interactions are also observable in citrus plants (Prasad et al.; Lin et al.). The destructive citrus disease, Huanglongbing, breaks out because in sap-sucking psyllids (Diaphorina citri) and Candidatus (Ca.) Liberibacter asiaticus infected citrus plants, the volatile cues are modified in a way that the $D$. citri predator, ladybird beetle $P$. japonica cannot find the host plant and reduce the insect pathogen, D. citri, population.

Plant health and productivity is of complex nature and strongly influenced by the intimate interaction of plants with deleterious and beneficial organisms. Obligate parasites affecting plant phloem such as $\mathrm{D}$. citri and Candidatus ( $\mathrm{Ca}$.) Liberibacter link cellular levels with interdisciplinary scales and in such systems also viruses play a key role as the tomato-whiteflyTomato Yellow Leaf Curl Virus (TYLCV) system studied by 
Tan et al.. Viral influence is also asserted by Li et al., who report that soil bensulfuron-methyl residues exhibit significant effects on the infestation of Bemisia tabaci, Myzus persicae, and Tobacco mosaic virus in Nicotiana tabacum and Trivedi et al. expanded such insights by highlighting the molecular, ecological, and evolutionary aspects of interactions among insects, plants, and their associated microbial communities. Islam et al., having nitrogen in their research focus, detail that a high level of nitrogen makes tomato plants release less volatiles and attract more Bemisia tabaci (Hemiptera: Aleyrodidae) and Santamaria et al. highlight that MATI proteins unveil a potential in defense through photosynthetic pigment modulation.

The Achnatherum sibiricum article of Qin et al. adds an aspect to the "Frontiers research topic Plant-Microbe-Insect Interaction: Source for Bio-fertilizers, Bio-medicines and Agent Research", worthwhile to have a closer look. They report in their e-book contribution that A. sibiricum, a little-studied, native grass of the Inner Mongolian steppe, is highly infected by endophytes but is not producing detectable endophyte-related alkaloids as known from agronomically important grasses such as tall fescue and perennial ryegrass. Despite the herbivore resistance, the

\section{REFERENCES}

Benckiser, G. (ed.). (1997). Fauna in Soil Ecosystems - Recycling Processes, Nutrient Fluxes, and Agricultural Production. New York, NY: Marcel Dekker, Inc.

Benckiser, G., and Schnell, S. (eds.). (2007). Biodiversity in Agricultural Production Systems. Boca Raton, FL: Taylor and Francis.

Conflict of Interest Statement: The authors declare that the research was conducted in the absence of any commercial or financial relationships that could be construed as a potential conflict of interest. resistance to hosting Locusta migratoria increases by mechanisms different from endophyte-conferred and alkaloid decline.

All the articles contributed to the research topic "PlantMicrobe-Insect Interaction: Source for Bio-fertilizers, Bio-medicines and Agent Research" reveal interesting interactive details, and show that our understanding of the complexity of agricultural production systems is only in its very beginning. Nevertheless, the given insights in soil properties-virus-microbeplant-insect interactions are hopefully a stimulus for continuing the discussion in this field.

\section{AUTHOR CONTRIBUTIONS}

All authors listed have made a substantial, direct and intellectual contribution to the work, and approved it for publication.

\section{ACKNOWLEDGMENTS}

We profusely thank the Editors of Frontiers in Plant Science for their continued help and support in successfully bringing out a Special Issue on the above Research Topic in their Journal and subsequently this e-Book.

Copyright (c) 2018 Benckiser, Hartmann, Kumar and Honermeier. This is an openaccess article distributed under the terms of the Creative Commons Attribution License (CC BY). The use, distribution or reproduction in other forums is permitted, provided the original author(s) and the copyright owner are credited and that the original publication in this journal is cited, in accordance with accepted academic practice. No use, distribution or reproduction is permitted which does not comply with these terms. 